\section{Review: possible benefits from early intensive behavioural and developmental interventions in children with autism spectrum disorders, but more research needed}

\section{QUESTION}

Question: Are early intensive behavioural and developmental interventions effective at enhancing developmental outcomes in children aged 12 and below with autism spectrum disorders (ASDs)?

Outcomes: Effects on core ASD symptoms and other symptoms commonly associated with ASDs, including cognitive functioning and behavioural challenges; harms/adverse events.

\section{METHODS}

Design: Systematic review

Data sources: MEDLINE, PsycINFO and ERIC were searched from 2000 to May 2010. Additional articles were identified through hand searches of reference lists of all included articles and review of clinical trials related to therapies for ASDs.

Study selection and analysis: All study designs which assessed early intensive behavioural and developmental interventions were eligible for inclusion, except for single case reports. Studies had to be published during or after 2000, involved 10 or more participants aged 12 years or younger with a diagnosis of ASD and could include studies with children younger than 2 who were at risk for ASD.

\section{MAIN RESULTS}

Of 34 studies met inclusion criteria, 23 were classified as UCLA/Lovaas-based interventions (EIBIs), 4 as comprehensive interventions for younger children and 7 as parent-training protocols. EIBI approaches: The EIBI studies included one randomised controlled trial (RCT), three non-RCT, five prospective cohorts, two retrospective cohorts and six prospective and six retrospective case series. Eight of these studies were of fair quality. Of these, the RCT compared EIBI with parent training and found that the EIBI group gained on average $15 \mathrm{IO}$ points compared with relatively stable $\mathrm{IO}$ in the parent-training group. IOs in both groups remained in the impaired range. The cohort studies and non-RCTs varied in the interventions and populations they assessed. They found varying results, with some studies showing improvements in outcomes such as IO, adaptive behaviour skills and symptoms with EIBI approaches compared with other interventions but other studies not finding differences. Overall, the strength of evidence for EIBI effects on language, cognitive, education and adaptive outcomes, and ASD symptom severity was low. Comprehensive approaches for children younger than 2 years old: There were four studies of comprehensive interventions for younger children: one good quality RCT, one fair quality nonRCT and two prospective case series. The RCT found that the Early Start Denver Model intervention provided significantly larger improvements in IQ and adaptive behaviour but not in symptom severity scores or repetitive behaviours, compared with a less-intensive intervention. These findings had not yet been replicated. The strength of evidence for comprehensive interventions for children below the age of 2 years was insufficient. Parent-training approaches: The studies investigating parent-training interventions included three RCTs, one nonRCT and three prospective case series. Three of the four comparative studies were of fair quality. The RCTs found some evidence of short-term improvements from parent training in certain areas, such as language, social and adaptive skills. Longterm outcomes were not assessed. Studies lacked standardised measures, and some studies had baseline differences between groups. The strength of evidence for an effect of parent-training on changing core ASD deficit areas was insufficient.

\section{CONCLUSIONS}

Studies have indicated that there may be benefits from early intensive behavioural and developmental interventions in children with ASD. However, there are limitations to the strength of the available evidence, and more research is needed. There is no sufficient evidence to determine which specific interventions are the most effective for treatment of children with ASDs.

\section{ABSTRACTED FROM \\ Warren Z, McPheeters ML, Sathe N, et al. A systematic review of early intensive intervention for autism spectrum disorders. Pediatrics 2011;127:e1303-11.}

Correspondence to Zachary Warren, Vanderbilt University, 230 Appleton Place/ PMB 74, Nashville, TN 37203-5721, USA; zachary.warren@vanderbilt.edu

Sources of funding Agency of Healthcare Research and Quality.

- References are available online at http://ebmh.bmj.com debate about the effectiveness of early interventions for young children with autism spectrum disorders (ASD). The earliest studies claimed that home-based behavioural programmes $(40 \mathrm{~h} /$ week for $\geq 2$ years beginning from age 2 ) could lead to significant improvements in 10 and even 'recovery from autism'1. The results of subsequent studies, however, have been inconsistent - some replicating these original claims and others reporting much more limited gains. This has resulted in a number of systematic reviews and meta-analyses ${ }^{2}$ with the overall conclusion that, although intensive behavioural interventions can be very effective for some children, the effects are far from universal. The poor quality of many of these trials is also highlighted. The present review, (which excludes some studies included in previous reviews but contains others previously rejected) replicates these general conclusions and stresses the need for more research into the characteristics of participants who do, or do not, respond to treatment.

In contrast to some other reviews, this review also includes interventions with a focus on early communication or general development, again reaching similar conclusions about individual differences in response to treatment. What is increasingly apparent is that treatment effects are relatively specific and circumscribed. They do not tend to reduce overall levels of autism severity.
In conclusion, because ASDs are highly heterogeneous conditions, not a treatment can be expected to work for all or to bring about 'recovery'. Identifying factors that moderate treatments' outcomes must be the goal of future research in this area. Although the task of identifying significant predictors of outcome will present many challenges, ${ }^{3}$ in these times of financial restraint, ensuring that treatments are focused and individually appropriate becomes even more essential.

\section{Patricia Howlin}

Department of Psychology, Institute of Psychiatry, London, UK

Competing interests None. 